\title{
ST2 as checkpoint target for colorectal cancer immunotherapy
}

\author{
Kevin Van der Jeught, ${ }^{1}$ Yifan Sun, ${ }^{1}$ Yuanzhang Fang, ${ }^{1}$ Zhuolong Zhou, ${ }^{1}$ Hua Jiang, ${ }^{2}$ Tao Yu, ${ }^{1}$ \\ Jinfeng Yang, ${ }^{2}$ Malgorzata M. Kamocka, ${ }^{3}$ Ka Man So, ${ }^{4}$ Yujing Li, ${ }^{1}$ Haniyeh Eyvani, ${ }^{1}$ \\ George E. Sandusky, ${ }^{5}$ Michael Frieden, ${ }^{1}$ Harald Braun, ${ }^{6,7}$ Rudi Beyaert, ${ }^{6,7}$ Xiaoming He, ${ }^{8,9}$ \\ Xinna Zhang, ${ }^{1,10}$ Chi Zhang, ${ }^{1,4,10}$ Sophie Paczesny, ${ }^{2,10}$ and Xiongbin Lu ${ }^{1,10}$ \\ 'Department of Medical and Molecular Genetics, 2Department of Pediatrics, ${ }^{3}$ Department of Medicine, Division of \\ Nephrology, ${ }^{4}$ Center for Computational Biology and Bioinformatics, ${ }^{5}$ Department of Pathology and Laboratory Medicine, \\ Indiana University School of Medicine, Indianapolis, Indiana, USA. ${ }^{6}$ VIB Center for Inflammation Research, Ghent, \\ Belgium. 'Department of Biomedical Molecular Biology, Ghent University, Ghent, Belgium. ${ }^{8}$ Fischell Department of \\ Bioengineering and ${ }^{9}$ Marlene and Stewart Greenebaum Comprehensive Cancer Center, University of Maryland, Baltimore, \\ Maryland, USA. ${ }^{10}$ Melvin and Bren Simon Cancer Center, Indiana University School of Medicine, Indianapolis, Indiana, USA.
}

Immune checkpoint blockade immunotherapy delivers promising clinical results in colorectal cancer (CRC). However, only a fraction of cancer patients develop durable responses. The tumor microenvironment (TME) negatively impacts tumor immunity and subsequently clinical outcomes. Therefore, there is a need to identify other checkpoint targets associated with the TME. Earlyonset factors secreted by stromal cells as well as tumor cells often help recruit immune cells to the TME, among which are alarmins such as IL-33. The only known receptor for IL-33 is stimulation 2 (ST2). Here we demonstrated that high ST2 expression is associated with poor survival and is correlated with low CD8 ${ }^{+} \mathrm{T}$ cell cytotoxicity in CRC patients. ST2 is particularly expressed in tumor-associated macrophages (TAMs). In preclinical models of CRC, we demonstrated that ST2expressing TAMs (ST2+ TAMs) were recruited into the tumor via CXCR3 expression and exacerbated the immunosuppressive TME; and that combination of ST2 depletion using ST2-KO mice with anti-programmed death 1 treatment resulted in profound growth inhibition of CRC. Finally, using the IL-33trap fusion protein, we suppressed CRC tumor growth and decreased tumor-infiltrating ST2+ TAMs. Together, our findings suggest that ST2 could serve as a potential checkpoint target for CRC immunotherapy.

Conflict of interest: SP has a patent, "Methods of detection of graft-versus-host disease" (US 20130115232A1, W0 2013066369A3), licensed to Viracor-IBT Laboratories.

Copyright: () 2020, American Society for Clinical Investigation.

Submitted: December 30, 2019

Accepted: April 8, 2020

Published: May 7, 2020.

Reference information: /CI Insight. 2020;5(9):e136073.

https://doi.org/10.1172/jci.

insight.136073.

\section{Introduction}

Colorectal cancer (CRC) ranks third among the most common cancers, affecting both men and women worldwide. Early detection of the disease is associated with better outcome. Unfortunately, it is often identified at a more advanced stage that remains challenging to treatment (1). The importance of the way in which tumor cells manipulate their environment to favor their growth and metastasis has become obvious (2). Cancer cells instruct stromal cells, such as cancer-associated fibroblasts, endothelial cells, adipocytes, tumor-associated macrophages (TAMs), along with other immune cells to orchestrate a "tolerogenic" tumor microenvironment (TME). Checkpoint immunotherapy has proven its significant efficacy for a subpopulation of patients harboring a high frequency of microsatellite instability (MSI-H) phenotype. However, the number of patients who benefit from these immunotherapies remains limited, as they currently apply in only about $5.9 \%$ of all CRC cases. Therefore, there is an unmet need to identify more checkpoint targets involving primary cell types and pathways in the TME $(3,4)$. At the early stage of tumorigenesis, several types of cells can release alarmin signals such as IL-33 in response to environmental stresses (5). The only known receptor binding the IL-33 is stimulation 2 (ST2). The IL-33/ST2 pathway has shown significant importance in CRC development and metastasis (5-8). Here, we evaluated the potential of the IL-33/ST2 pathway to serve as a checkpoint target for CRC immunotherapy. 


\section{Results}

ST2 expression on macrophages is associated with low $C D 8^{+} T$ cell cytotoxicity. To determine the clinical relevance of ST2 in CRC, we generated a Kaplan-Meier using a combination of the GSE41258 ( $n=165$ CRC patients) and the GSE39582 $(n=505)$ databases. Decreased survival was observed in patients with high ST2 (IL-1 receptor-like 1 [IL1RL1]) gene expression, as determined by comparing ILIRL $1^{10}$ and $I L 1 R L 1^{h t}$ populations (Figure 1A). To identify the cells in the TME that highly express ST2, we determined normalized ST2 expression in a variety of cell types present in the TME and found that macrophages expressed ST2 to a higher degree than other cell types (Figure 1B). We validated abundant expression of ST2 in macrophages using confocal microscopy on stage I-IV CRC tumor tissues from the Indiana University Simon Cancer Center Tissue Bank (Figure 1, C and D, and Supplemental Table 1; supplemental material available online with this article; https://doi.org/10.1172/jci.insight.136073DS1). Next, we used the inference of cell types and deconvolution (ICTD) algorithm to assess the correlation of ST2 expression with the relative $\mathrm{CD}^{+} \mathrm{T}$ cell cytotoxicity (9). This method allows for an unbiased inference of cell proportions and activity from bulk tissue RNA-Seq data. We found negative correlation between ST2 expression and $\mathrm{CD}^{+} \mathrm{T}$ cell cytotoxicity, while no significant changes were observed in the tumor infiltration of total $\mathrm{T}$ cells between ST2-high and -low cohorts (Figure 1E and Supplemental Figure 1). These data indicate the potential functional role of ST2 ${ }^{+}$TAMs and indicate that further investigation of the IL-33/ST2 pathway in CRC is warranted (3).

Disruption of the IL-33/ST2 pathway enhances CD8 $T$ cell-mediated antitumor responses. We first assessed mouse survival and the growth of CRC tumors in immunocompetent ST2-KO (ST2-/) mice (Figure 2, A and B, and Supplemental Figure 2). Growth of MC38 and CT26 tumors was much slower in $S T 2^{-/-}$mice compared with WT control mice. As expected, similar tumor growth inhibition was observed in male and female $S T 2^{-1-}$ mice (Supplemental Figure 2B). Because of an inverse correlation between ST2 expression and $\mathrm{CD}^{+} \mathrm{T}$ cell cytotoxicity, we wanted to examine the ST2-associated immunological changes in the TME. To this end, we profiled MC38 tumors from WT and $S T 2^{-/-}$mice using a 27-marker antibody panel for mass cytometry (CyTOF). A SPADE on viSNE single-cell dimensional analysis was conducted to assess immune cell profiles. Enhanced $\mathrm{CD} 8^{+} \mathrm{T}$ cell infiltration was observed in the $S T 2^{-{ }^{-}}$mice and validated by immunohistochemical staining of tumor samples, whereas other immune cells were not significantly impacted (Figure 2, C and D, and Supplemental Figure 3). Furthermore, host ST2 depletion alleviated $\mathrm{CD}^{+} \mathrm{T}$ cell exhaustion, as exemplified by lower lymphocyte activation gene 3 (Lag3) expression (Figure 2, $\mathrm{E}$ and $\mathrm{F})(10,11)$. To confirm the central role of $\mathrm{CD} 8^{+} \mathrm{T}$ cells in the observed antitumor effects, we depleted $\mathrm{CD}^{+} \mathrm{T}$ cells from the tumor-bearing mice and showed that depletion of $\mathrm{CD}^{+} \mathrm{T}$ cells abolished the tumor-inhibiting effects of $S T 2^{-/-}$. These results suggested that IL-33/ST2 signaling acts on the capacity of $\mathrm{CD}^{+} \mathrm{T}$ cells to mediate antitumor immunity (Figure 2, G and $\mathrm{H}$, and Supplemental Figure 4).

ST2-expressing macrophages exert T cell immunosuppression. Because the human data analysis revealed predominant expression of ST2 on macrophages, we next evaluated expression of ST2 in distinct immune cell populations from MC38- and CT26-derived tumors in C57BL/6 and BALB/c mice, respectively (Figure 3, A and B, and Supplemental Figures 3, 5, and 6). Our analysis identified expression of ST2 in various types of immune cells, including Tregs (Figure 3A) (7, 8, 12-14). Once normalized to the frequency of each type of cells present in the TME, only TAMs showed marked infiltration in these CRC mouse models (Figure 3, A and B). We observed an increased number of ST2 ${ }^{+}$TAMs during tumor progression (Figure 3C). The presence of ST2 TAMs was confirmed in the orthotopic cecal wall model (Figure 3, D and E, and Supplemental Figure 6). No $\mathrm{ST}^{+}$TAMs were identified in the healthy cecum (Figure 3E and Supplemental Figure 6B). To understand the mechanism by which ST2 ${ }^{+}$TAMs exhaust $\mathrm{CD}^{+} \mathrm{T}$ cells, we first looked at the polarization of ST2 ${ }^{+} \mathrm{TAMs}$. The M2 macrophage activation profile of the ST2 ${ }^{+}$TAMs in our tumor models displayed high macrophage mannose receptor (MMR, CD206) and low MHC class II expression (Figure 4A). These results are in line with a previous study demonstrating the role of the IL-33/ST2 axis in cancer metastasis $(15,16)$. Further assessment of the macrophage phenotype revealed that ST2 ${ }^{+}$TAMs displayed a Ly $6 \mathrm{C}^{\mathrm{lo}} \mathrm{Ly} 6 \mathrm{G}^{\mathrm{lo}} \mathrm{CD} 169^{\text {hi }}$ Sirpa $\mathrm{a}^{\text {hi }}$ phenotype, which was shared by ST2 ${ }^{+}$TAMs at early $\left(330 \pm 37.7 \mathrm{~mm}^{3}\right)$ and later tumor stages $\left(1066 \pm 183.7 \mathrm{~mm}^{3}\right)$ (Figure $3 \mathrm{C}$, Figure 4B, and Supplemental Figure 7). Moreover, ST2 ${ }^{+}$TAMs at the initiation of tumor development did not express CXCR3, while ST2 ${ }^{+}$TAMs at the late stage expressed high levels of this marker, suggesting an infiltration from the periphery (Figure 4C). CXCR3-expressing macrophages have been reported to promote tumor dissemination (17-20). To functionally identify the suppressive capacity of ST2 ${ }^{+} \mathrm{TAMs}$, we conducted a coculture experiment with TAMs and T cells (Supplemental Figure 8, A and B). The results demonstrated the 
A

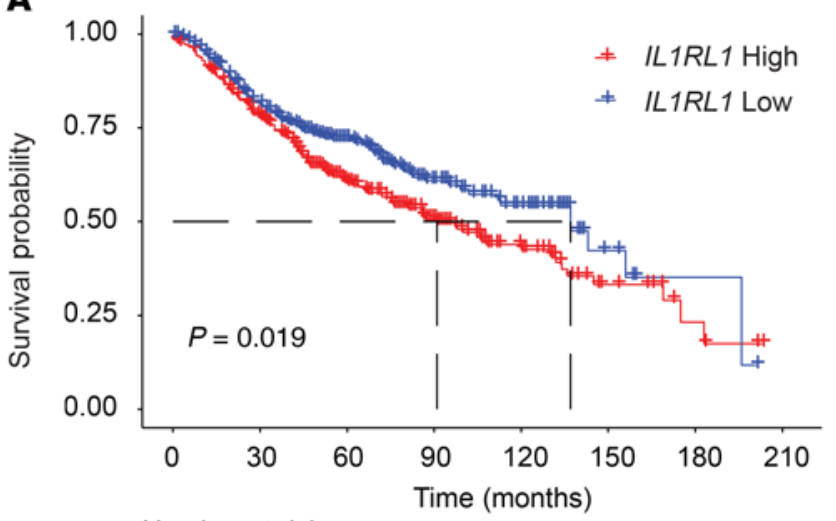

Number at risk

蛋 - \begin{tabular}{cccccccc}
299 & 211 & 124 & 64 & 32 & 12 & 4 & 0 \\
297 & 218 & 141 & 63 & 31 & 7 & 3 & 0 \\
\hline 0 & 30 & 60 & 90 & 120 & 150 & 180 & 210 \\
Time (months)
\end{tabular}

B

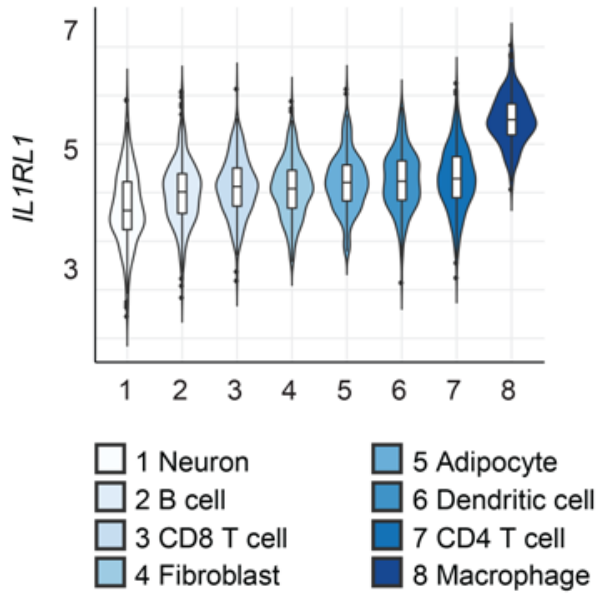

C MERGE

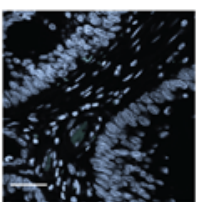

TUMOR

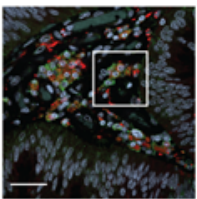

INSET

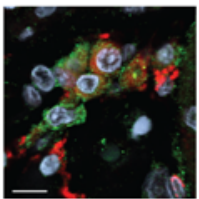

DAPI
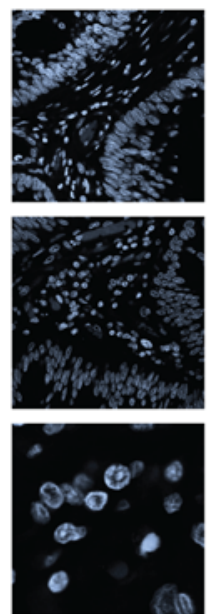

ST2
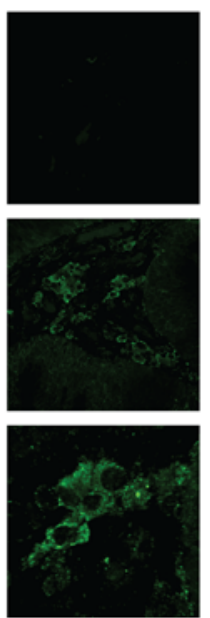

CD68
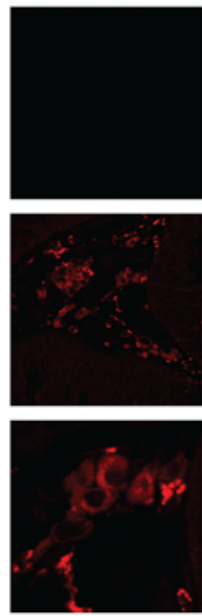

D ST2 ${ }^{-}$TAM

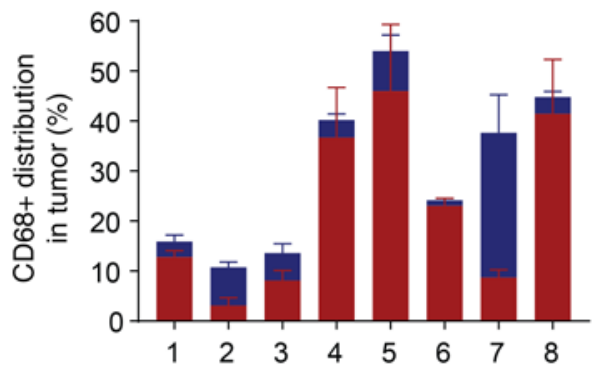

Patient \#

IL1RL1 Low

IL1RL1 High

E

Relative

CD8A

SLA2

NKG7

GZMH
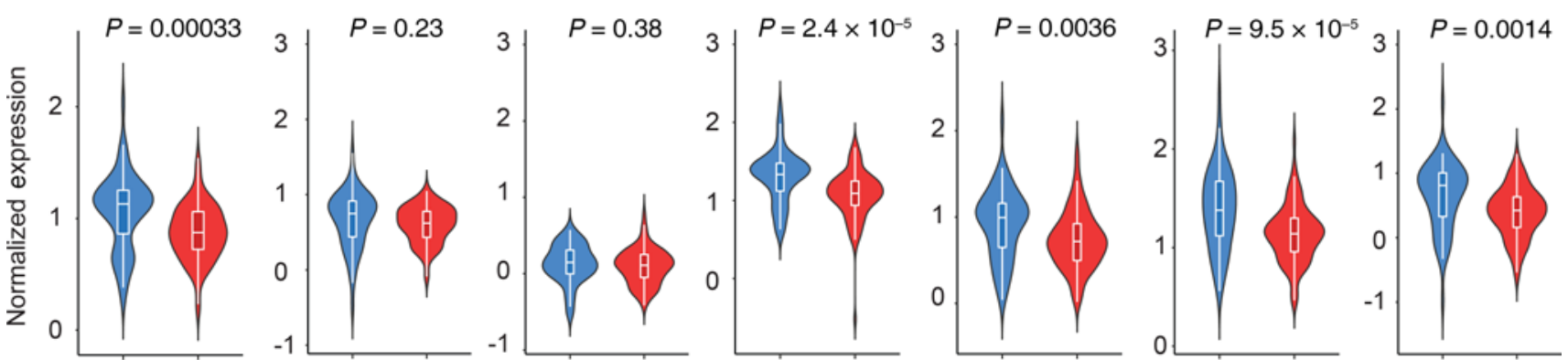

Figure 1. Identification of ST2 as a T cell-suppressive molecule in human CRC. (A) Kaplan-Meier survival curve from the combination of the GSE41258 ( $n=165$ ) and GSE39582 ( $n=505$ ) data sets of CRC patients with high and low IL1RL1 expression (top and bottom 40\%). (B) Normalized expression of IL1RL1 for the indicated cell types. The data were obtained from a large collection of microarray data as described in Methods. (C) Representative confocal images of ST2 expression on formalin-fixed, paraffin-embedded sections from CRC patients (stages I-IV) listed in Supplemental Table 1. ST2 is visualized in green, CD68 in red. Nuclei were counterstained with DAPI and visualized in gray. Secondary antibodies only were used as a negative control (NC). Scale bars: $40 \mu \mathrm{m}, 10 \mu \mathrm{m}$ (inset). (D) For each patient, a set of 4-7 images was taken throughout the entire tumor section to calculate the number of CD68 ${ }^{+}$cells and their distribution of ST2. Quantification of percentages was done after training the Imaris software mask to avoid any bias. (E) Violin box plots for the correlation of IL1RL1 (ST2) gene expression with relative T cell cytotoxicity (CD8A, SLA2, NKG7, PRF1, GZMA, and CZMH) and with each of the markers. Data were obtained analyzed using the ICTD algorithm on 93 biologically independent CRC patients with T cell infiltration (IL1RL1-high, 53 patients; IL1RL1-low, 40 patients) from the TCGA database. Significance was determined by log-rank test (A) and 2-tailed unpaired $t$ test (B). 
superior capacity of ST2 ${ }^{+}$TAMs as compared with ST2- TAMs to suppress T cell proliferation and IFN- $\gamma$ secretion (Figure 4, D and E, and Supplemental Figure 8C). To confirm in vivo the inhibitory role of ST2 ${ }^{+}$TAMs in $\mathrm{CD}^{+} \mathrm{T}$ cell-mediated immune responses, we grew MC38-derived tumors in $\mathrm{WT}$ and $\mathrm{ST2}^{-1}$ mice, and depleted TAMs with neutralizing anti-mouse CSF1R antibody (Figure 4, F and G, and Supplemental Figure 8D). There was no difference in tumor growth in those TAM-depleted mice, thus confirming the functional role of ST2 TAMs in tumor progression.

Enhanced responsiveness to anti-PD-1 therapy upon disruption of the IL-33/ST2 pathway. We further evaluated the potential of targeting the IL-33/ST2 pathway to enhance antigen-specific immune responses. To this end, CD8-enriched OT-I T cells, carrying a T cell receptor specific for the OVA epitope SIINFEKL, were transferred into naive WT and $S T 2^{-1-}$ mice. The mice were then immunized with the SIINFEKL peptide together with the adjuvant polyinosinic-polycytidylic acid [poly(I:C)]. Expansion of OT-I CD8 ${ }^{+} \mathrm{T}$ cells from the spleen was evaluated on day 5 (Figure 5A and Supplemental Figure 9A). Significantly more antigen-specific $\mathrm{CD}^{+} \mathrm{T}$ cells were detected in $S T 2^{-/-}$mice. We showed that upon specific peptide restimulation, these dextramer-positive $\mathrm{CD}^{+} \mathrm{T}$ cells were fully capable of secreting IFN- $\gamma$ and TNF- $\alpha$ (Figure 5B). Both WT and $S T 2^{-/-}$mice displayed antigen-specific $\mathrm{CD}^{+} \mathrm{T}$ cells with high expression levels of programmed cell death protein 1 (PD-1)(Figure 5C). These data indicate the potential benefit of combining IL-33/ST2 pathway blockade with anti-PD-1 treatment. To test the therapeutic potential of ST2 blockade, we treated MC38 tumor-bearing WT and $S T 2^{-/-}$mice with anti-PD-1 antibody. We demonstrated that anti-PD-1 treatment of $S T 2^{-1-}$ mice depleted of ST2 in the TME resulted in significantly greater tumor inhibition compared with treatment of WT mice (Figure 5D).

Inhibition of the IL-33/ST2 pathway enhances antigen-specific T cell responses in vivo. It was previously shown in the context of Tregs that coculture with IL-33 drives ST2 expression (6-8). The IL-33/ST2 pathway thus has the intrinsic capacity to regulate itself in a positive feedback loop. We reasoned that IL-33 also drives ST2 expression on the TAMs in our CRC mouse models. Therefore, we isolated TAMs from the MC38 tumor-bearing mice and cultured them in the presence of IL-33. Exposure to IL-33 enhanced ST2 expression in a dose-dependent manner (Figure 5, E and F, and Supplemental Figure 9B). We then tested whether we could alter the ST2 ${ }^{+}$TAM population in vivo by targeting the IL-33/ST2 pathway using the IL-33trap fusion protein, which consists of the extracellular parts of ST2 and its coreceptor IL-1 receptor accessory protein (IL-1RAcP) — fused by a flexible linker — and is able to capture free IL-33 with high affinity (Figure 5G) (21). Administration of IL-33trap from the onset of tumor development resulted in significantly reduced tumor growth as compared with vehicle treatment (Figure $5 \mathrm{H}$ ). No significant changes in body weight were observed, and histological analyses showed no toxic side effects from the IL-33trap treatment (Supplemental Figure 10, A and B). Analysis of the TME of control and IL-33trap-treated mice revealed a significant reduction in ST2 ${ }^{+}$TAM distribution (Figure $5 \mathrm{I}$ ). There were no significant changes in other immune cells analyzed in the study (Supplemental Figure 10C). These results suggest that interference with IL-33 can alter in vivo TAMs and their ST2 expression, leading to antitumor effects.

\section{Discussion}

The realm of checkpoint cancer immunotherapy has expanded dramatically since the discovery of cytotoxic T lymphocyte-associated protein 4 (CTLA-4) and PD-1 $(22,23)$. A mainstream area of research is focused on how to improve these established checkpoint targets and to predict which patients might benefit from these treatments (24). The unmatched power of cancer checkpoint immunotherapy to treat so-far-incurable tumors has prompted the discovery of additional checkpoint targets and pathways (25). These are particularly of interest as they provide (i) an alternative for initial resistant patients and (ii) superior, dual checkpoint blockade, thereby improving current checkpoint targets $(3,24)$. The major targets have been identified to date through a direct focus on the cells mediating tumor killing, such as $\mathrm{T}$ cells, including PD-1, T cell immunoglobulin, and mucin domain-containing protein 3 (Tim3) and Lag3 (24). However, limited efforts have been made to identify checkpoint targets on other cell types. Recently, Siglec-15 has been identified as an additional immune checkpoint on TAMs, showing promising results in preclinical mouse models, and is now being tested in a phase I clinical trial (NCT03665285) (26). In this study, we report the potential of ST2 as a therapeutic checkpoint target for CRC immunotherapy.

In $\mathrm{CRC}$, initial studies using nivolumab (PD-1) showed no significant responses in unselected patients. Since then, a small subset of patients (5.9\%) with an MSI-H phenotype meet the criteria to be enrolled for checkpoint immunotherapy (27-30). However, the checkpoint immunotherapy is currently 
A

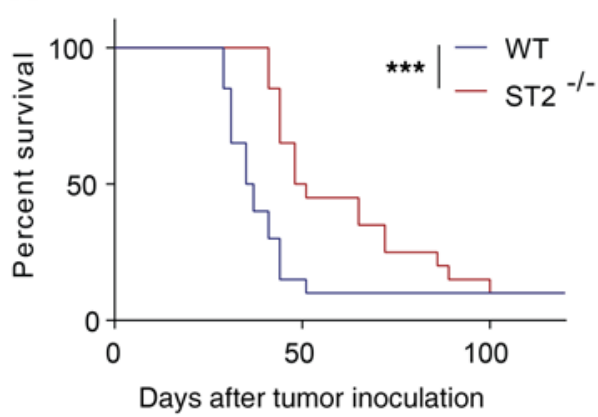

B

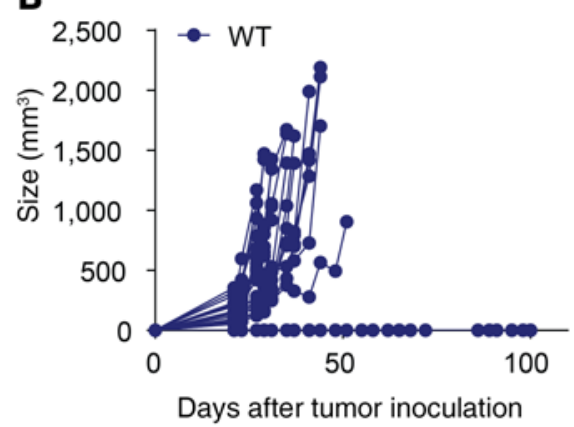

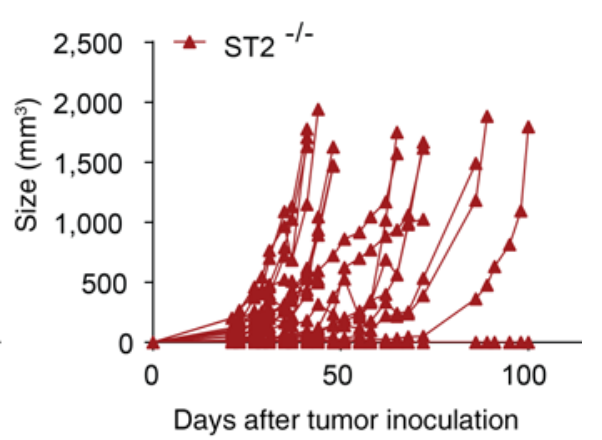

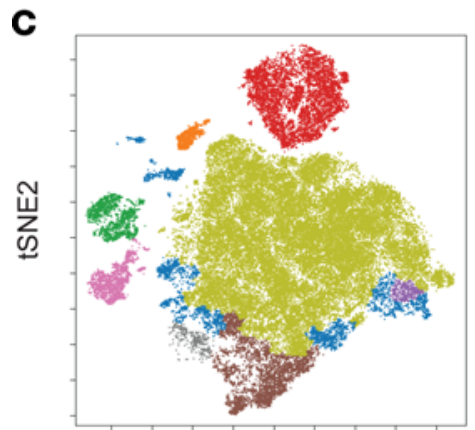

tSNE1

D

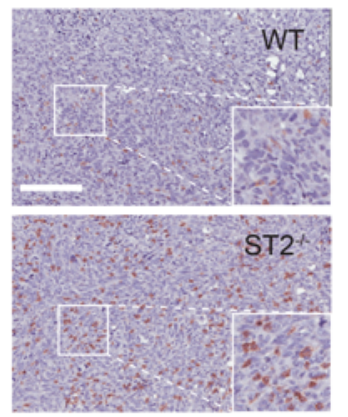

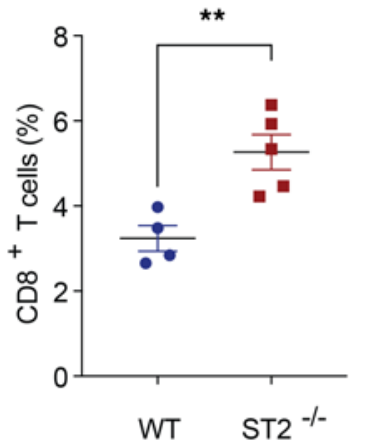

WT $\mathrm{ST} 2^{-}$

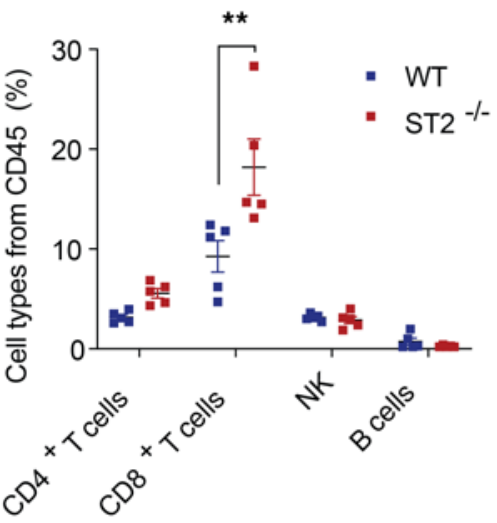

E

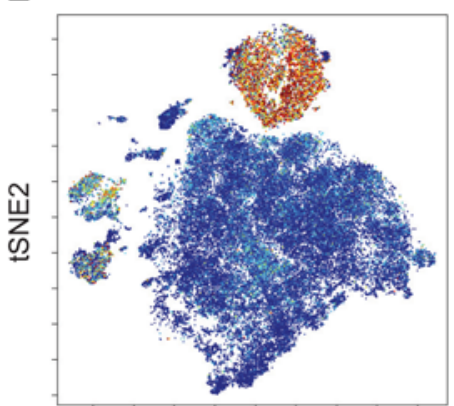

tSNE1
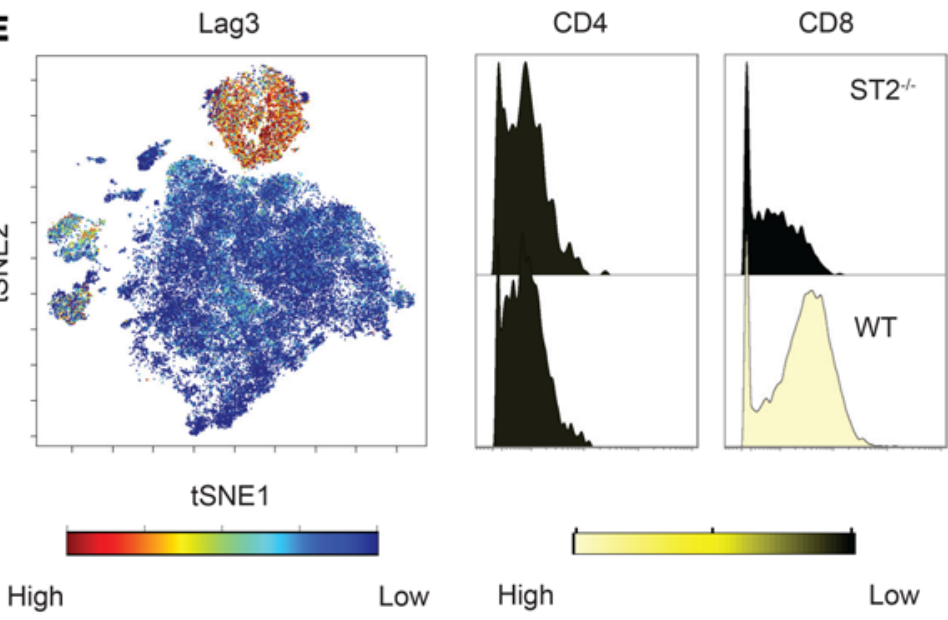
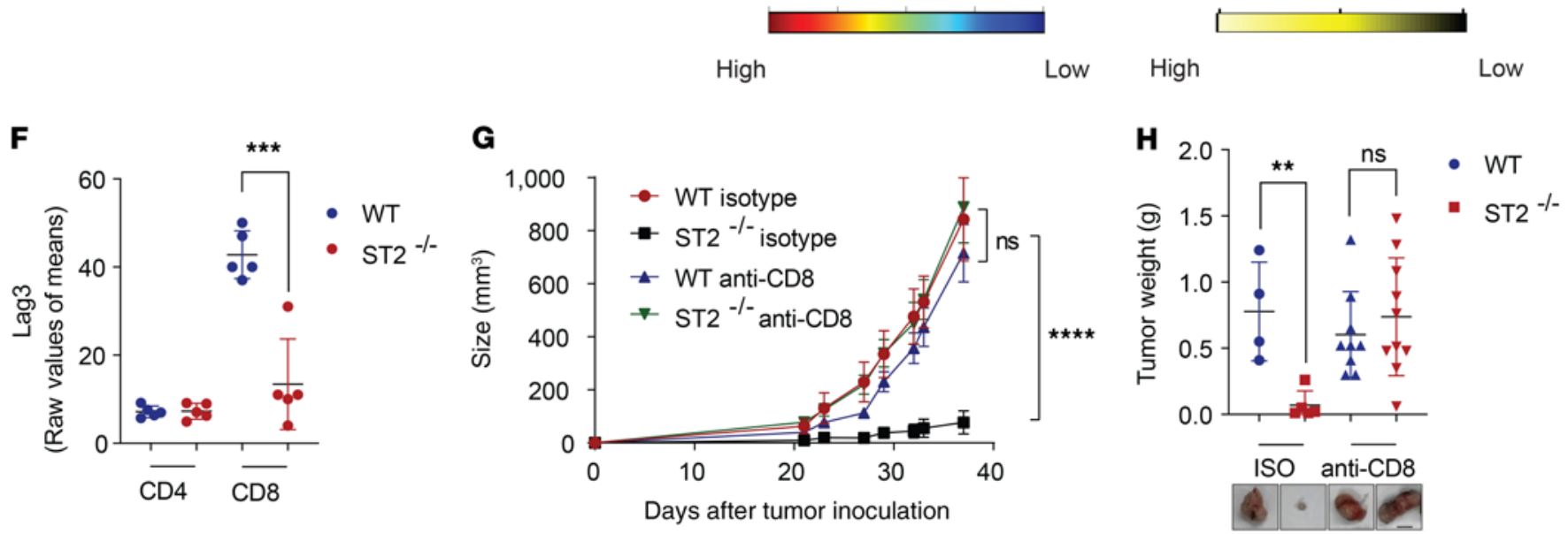

Figure 2. Disruption of the IL-33/ST2 pathway enhances CD8 ${ }^{+}$T cell-mediated antitumor responses. Survival (A) and tumor growth (B) of indicated MC38 CRC-engrafted mice (WT, $n=20 ; 5 T 2^{-1-}, n=20$ ). (C) viSNE representation of the immune cell subsets after SPADE clustering and quantification of the cell populations. Analysis of the TME from MC38 tumors using a 27-marker CyTOF panel (WT, $n=5 ; 5 T 2^{-1-}, n=5 ;$ data displayed as mean + SEM). (D) CD8 expression visualized in brown from WT and ST2 $2^{-1-}$ MC38 tumors (WT, $n=4 ; S T 2^{-1,}, n=5$ ). Scale bar: $100 \mu \mathrm{m}$. (E and F) Analysis for Lag3 exhaustion on CD4+ and CD8+ T cell subsets (WT, $n=5 ; S T 2^{-/-}, n=5$; data displayed as mean + SD). Growth (G) and weight (H) of 
MC38 tumors upon CD8 depletion (WT isotype, $n=4$; ST2 ${ }^{-/-}$isotype, $n=5$; WT anti-CD8, $n=9$; ST2 ${ }^{-/-}$anti-CD8, $n=10$; data displayed as mean + SEM). Scale bar: $1 \mathrm{~cm} .{ }^{* *} P<0.01,{ }^{* *} P<0.001,{ }^{* * * *} P<0.0001$. Significance was determined by log-rank test $(\mathbf{A}), 2$-tailed unpaired $t$ test $(\mathbf{C}$, D, F, and $\mathbf{H})$, and 2-way ANOVA (G).

effective only in a minority of patients, and not every MSI-H patient responds well, supporting the urgent need to identify other checkpoint targets associated with the TME. CRC is a particularly inflammatory cancer due to its intrinsic mucosal environment. Therefore, we investigated the potential role of the alarmin signal IL-33 and its concurrent IL-33/ST2 pathway for CRC immunotherapy. We identified in human CRC databases that ST2 expression is correlated with low CD8 ${ }^{+} \mathrm{T}$ cell cytotoxicity and poor patient survival. Furthermore, we identified that a significant number of CRC patients had ST2 ${ }^{+}$TAMs. A role for $\mathrm{ST}^{+}$TAMs in cancer metastasis has been recently described (15). To characterize these ST2 ${ }^{+}$ TAMs, we first identified their presence and phenotype in MC38 (MSI-H) and CT26 (MSS) preclinical models (31). While no ST2 ${ }^{+}$TAMs were found in normal stroma, an increased number of ST2 ${ }^{+}$TAMs were present within the tumor stroma of orthotopically injected tumors. In addition, our data suggest that the ST2 ${ }^{+}$TAMs have both phenotypic and functional immune suppression features (3). In a different tumor model, as reported by Andersson et al., genome-wide expression profiling of IL-33-stimulated TAMs confirmed their M2 phenotype (15). Further characterization of ST2 ${ }^{+}$TAMs revealed that their tumor infiltration is in part mediated via the CXCR3 axis, as at later time point ST2 ${ }^{+}$TAMs upregulate CXCR3. Aside from the CXCR3 pathway described herein, activation of the IL-33/ST2 pathway has been reported to promote macrophage infiltration in tumors of IL-33-transgenic mice overexpressing IL-33 (32, 33). Importantly, we showed that in our CRC models, ST2 is not directly expressed in the $\mathrm{CD}^{+} \mathrm{T}$ cells, although previous studies showed low levels of ST2 expression in CD8 ${ }^{+} \mathrm{T}$ cells in different types of cancer (34-36). Depletion of ST2 in $S T 2^{-{ }^{-}}$mice increased intratumor $\mathrm{CD}^{+} \mathrm{T}$ cell frequencies and function, and acted synergistically with anti-PD-1 checkpoint blockade for treating colorectal tumors. Even though we provide evidence that the main cell population expressing ST2 in the TME of our models are TAMs, we cannot rule out the possible effect on other cells using total body $S T 2^{-/-}$mice. It would be of interest to generate conditional ST2-knockout models to confirm these findings.

We identified that IL-33, which is also abundantly present in the TME, can drive ST2 expression on TAMs. Neutralization of IL-33 in the TME using IL-33trap was proven to be efficient in suppressing the $\mathrm{ST}_{2}{ }^{+} \mathrm{TAM}$ distribution. However, several limitations remain in therapeutically targeting the IL-33/ST2 pathway for cancer treatment using the fusion protein IL-33trap (extracellular parts of ST2 and IL-1RAcP fused by a flexible linker). Even though, IL-33trap is superior to soluble ST2 (sST2) in sequestering IL-33, its half-life requires daily treatment (21). Other than IL-33, IL-4 and IL-13 are also capable of regulating ST2 expression on macrophages $(37,38)$. For translational purposes, several options are under development to target the IL-33/ST2 pathway therapeutically. Previously, an ST2 inhibitor (small molecule) has been used in the context of graft-versus-host disease (39). The generation of neutralizing antibodies would be of importance, as they can be produced in higher amounts and would have a longer half-life. Genentech has developed an ST2 antibody (MSTT1041A) for inflammatory diseases such as chronic obstructive pulmonary disease, which is currently tested in a phase II clinical trial (NCT03615040). Studying T cell functionality upon therapeutic disruption of the IL-33/ST2 pathway with anti-ST2 antibodies would provide insight into the degree of recovery of exhaustion (e.g., enhanced capacity to secrete IFN- $\gamma$ and TNF- $\alpha$; reduced expression of Tim3, Lag3, and PD-1) after inhibition of this pathway. Combination of these approaches together with current checkpoint immunotherapies such as anti-PD-1 or anti-CTLA-4 antibodies would provide valuable insight. In addition, combination of therapeutic vaccination strategies such as dendritic cell vaccination using self-antigens, together with IL-33/ST2 blockade in cancer models, would provide essential insight toward further clinical development (40).

We conclude that ST2 could serve as an additional immunotherapy checkpoint in CRC. In addition, this study highlights the potential to target non-T cell subsets, which helps identify additional immune checkpoints in the TME and enhance therapeutic efficacy of immune checkpoint blockade therapy. ST2 ${ }^{+}$TAMs and their associated clinical features could serve as a prognostic markers in CRC immunotherapy. We therefore suggest the further development of ST2 as therapeutic target for CRC immunotherapy. 
A

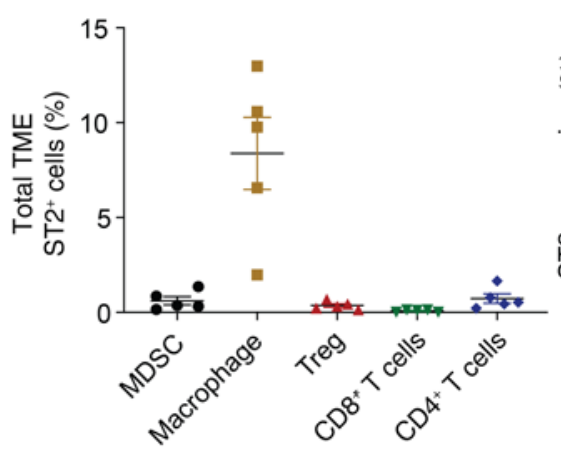

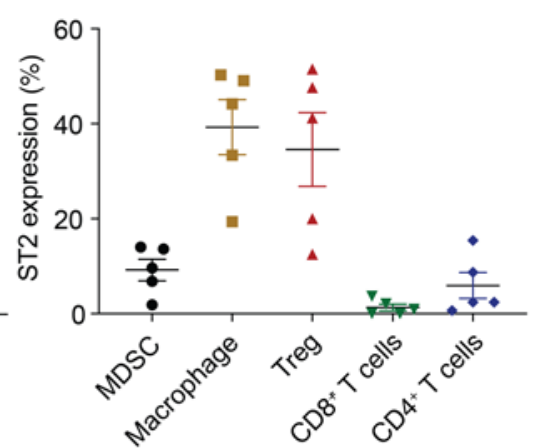
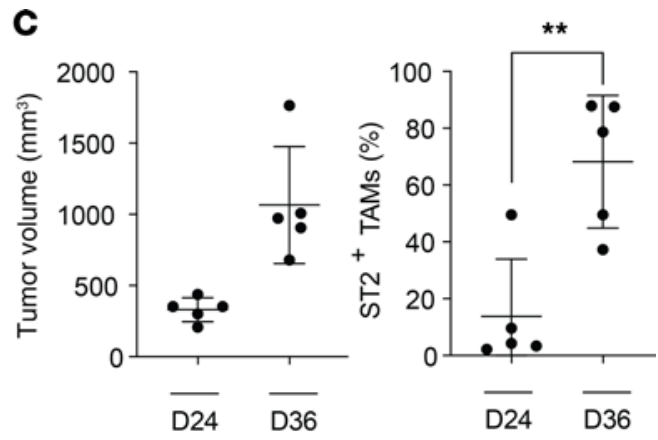

B

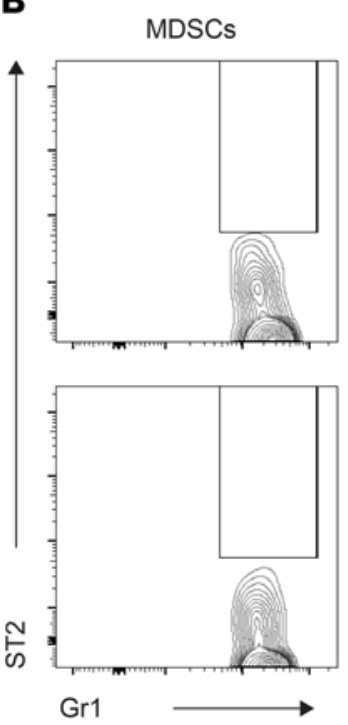

D

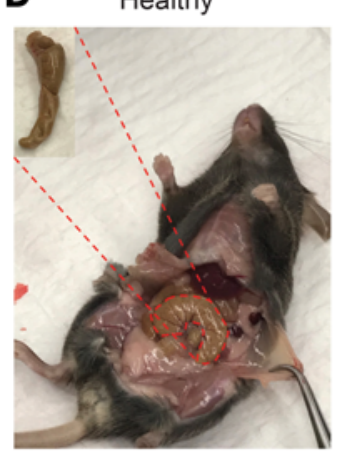

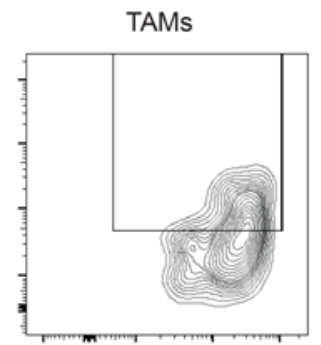

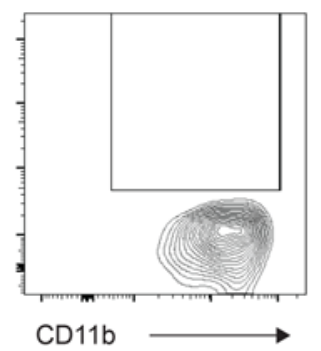

Tumor

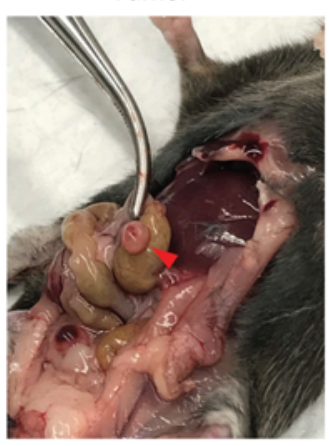

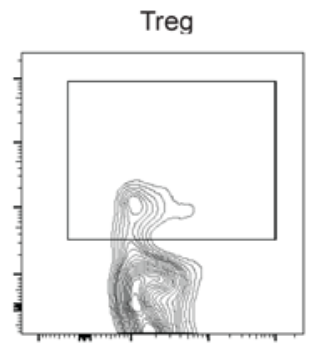
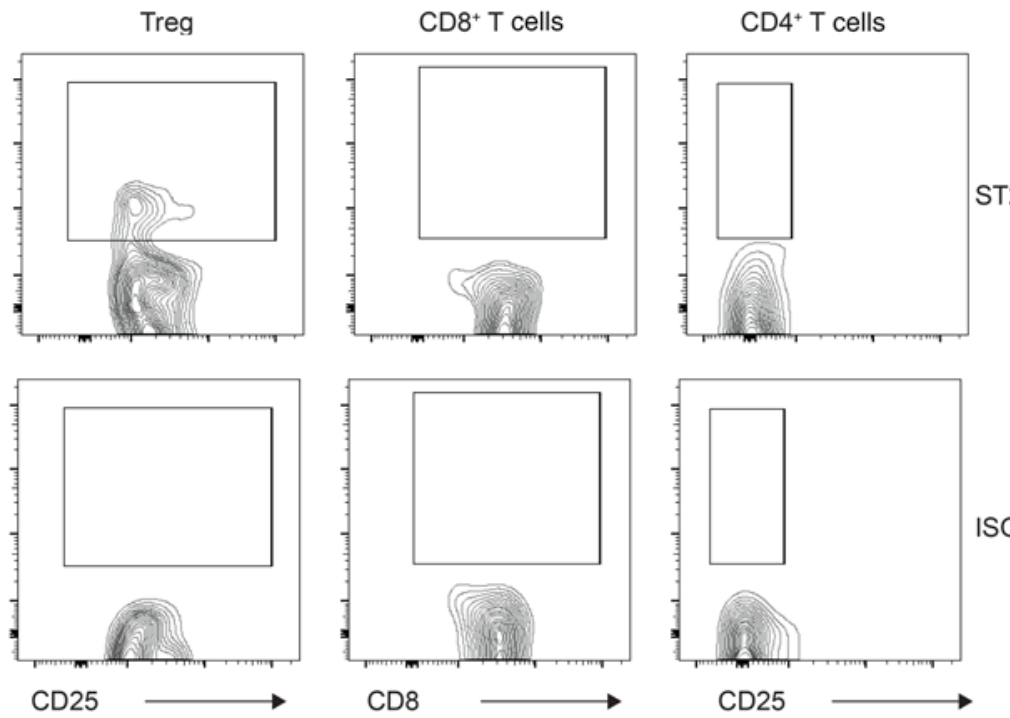

E
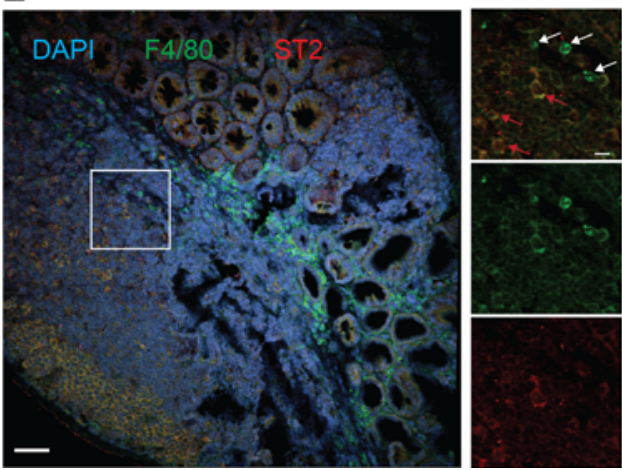

Figure 3. Identification of ST2 ${ }^{+}$TAMs in preclinical mouse models. (A) Total ST2+ immune cells found within the CT26 TME (CD45 ${ }^{+}$population); and distribution of $\mathrm{ST}_{2}+$ percentages among the indicated cell types $(n=5$; data displayed as mean $+\mathrm{SEM})$. (B) Representative flow cytometry plots from $\mathbf{A}$ for determining ST2 expression in distinct immune cell subsets. (C) Infiltration of ST2+ TAMs in MC38 tumors at an early stage (day 24) and late stage of tumor growth (day 36). Tumor volume and quantification of ST2 ${ }^{+}$TAMs in MC38 tumors of mice sacrificed at the indicated time points ( $n=5$; data displayed as mean + SD). (D) Image of MC38 orthotopic cecal wall tumor. (E) Identification of ST2+ TAMs in the orthotopic cecal wall of MC38 injected tumor model. Representative confocal microscopy images. In the inset, white arrows indicate ST2- TAMs, and red arrows indicate ST2 ${ }^{+}$TAMs $(n=5)$. Scale bar: 50 $\mu \mathrm{m}, 15 \mu \mathrm{m}$ (inset). Quantification of ST2 $2^{+}$TAM percentages in normal stroma, tumor stroma, and tumor $(n=3)$. ${ }^{*} P<0.01$. Significance was determined by 2-tailed unpaired $t$ test (C, right panel). 
A

ST2

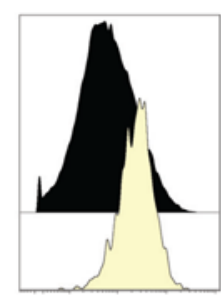

CD206

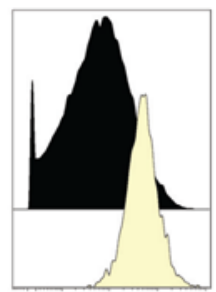

MHC II (IA-IE)

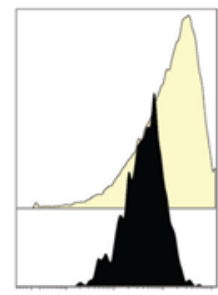

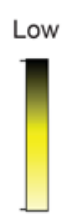

High
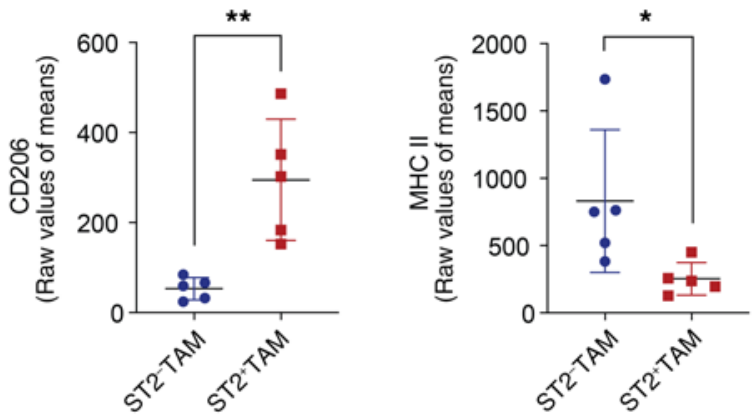

B

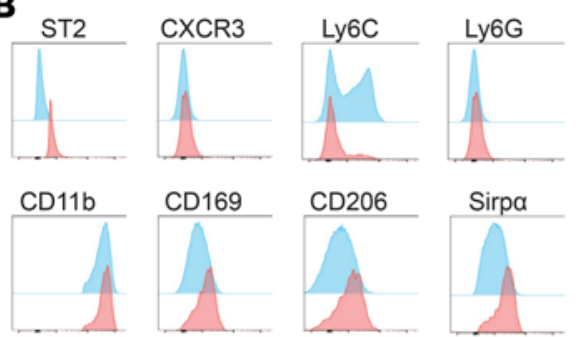

ST2-TAM

ST2+TAM
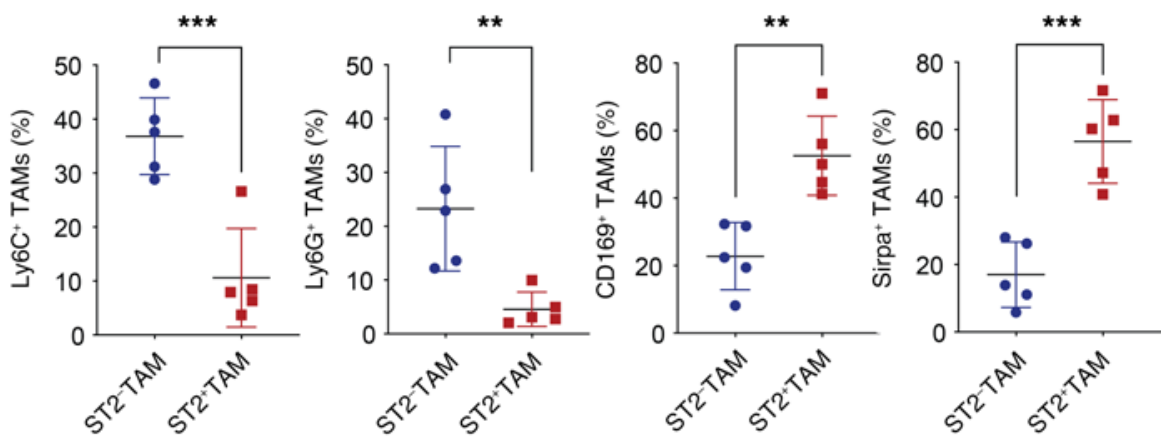

C
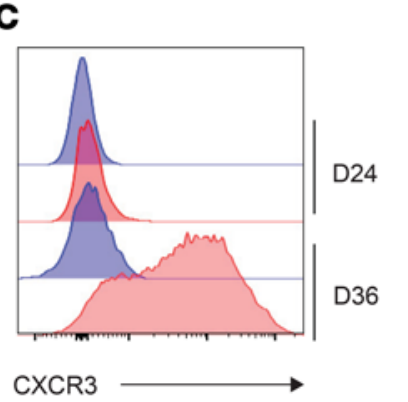

D

- ST2-TAM

- ST2*TAM

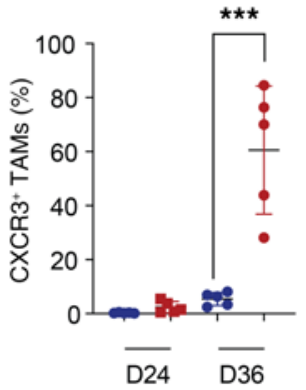

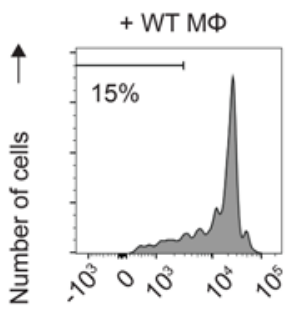

CFSE

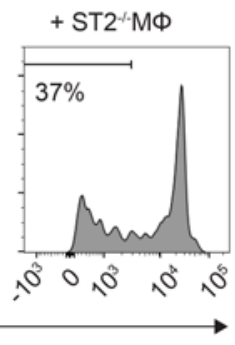

CD8

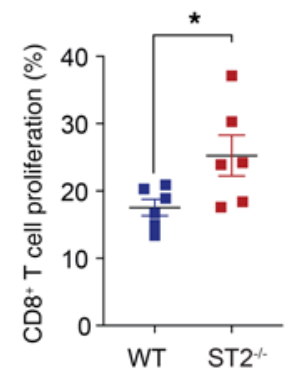

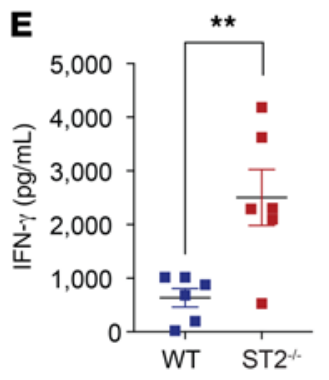

F

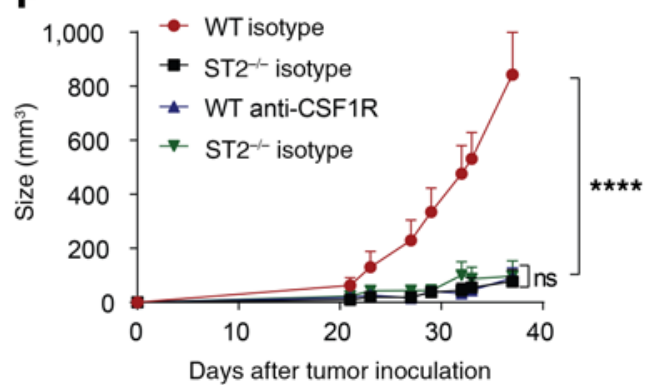

G

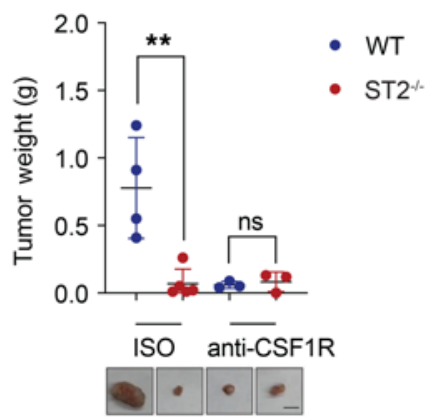

Figure 4. ST2 ${ }^{+}$TAM characterization. (A) CyTOF analysis of M2 phenotype of ST2 ${ }^{+}$TAMs and ST2- TAMs from MC38 tumors. Representative CyTOF overlay histograms; and quantification ( $n=5$; data displayed as mean + SD). (B) Flow cytometry analysis of ST2+ TAMs and ST2- TAMs from MC38 tumors. Representative histogram overlays of ST2 ${ }^{+}$TAMs and ST2- TAMs for the indicated surface markers; and quantification ( $n=5$; data displayed as mean + SD). (C) Expression levels of CXCR3 on ST2 ${ }^{+}$TAMs during tumor development. Representative histogram of CXCR3 expression and quantification ( $n=5$; data displayed as mean + SD). (D) Macrophage suppression assay of MC38-enriched macrophages from WT and ST2 ${ }^{-1-}$ mice. Representative flow cytometry histograms of CD8 ${ }^{+}$T cell proliferation (CFSE) upon distinct coculture conditions; and quantification of CD8 ${ }^{+}$T cell proliferation upon TAM coculture ( $n=6$; data displayed as mean + SEM). (E) Quantification of T cell IFN- $\gamma$ secretion on supernatants upon TAM coculture $(n=6$; data displayed as mean + SEM). Growth (F) and weight (G) of MC38 tumors upon macrophage depletion using anti-CSF1R antibodies (WT isotype, $n=4 ;$ ST2 $2^{-1-}$ isotype, $n=5$; WT anti-CSF1R, $n=6 ; S T 2^{-1-}$ anti-CSF1R, $\left.n=6\right)\left(\mathbf{F}\right.$, data displayed as mean + SEM; G, mean + SD) Scale bar: $1 \mathrm{~cm} .{ }^{*} P<0.05,{ }^{* *} P<0.01,{ }^{* * *} P<0.001,{ }^{* * * *} P<0.0001$. Significance was determined by 2-tailed unpaired $t$ test (A-E) and 2-way ANOVA (F). 
A

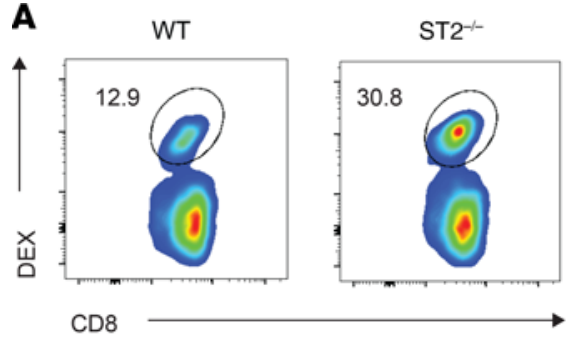

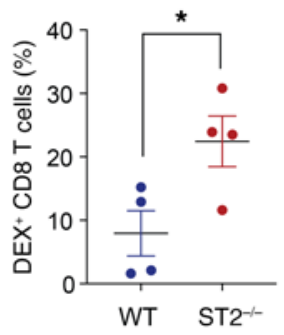
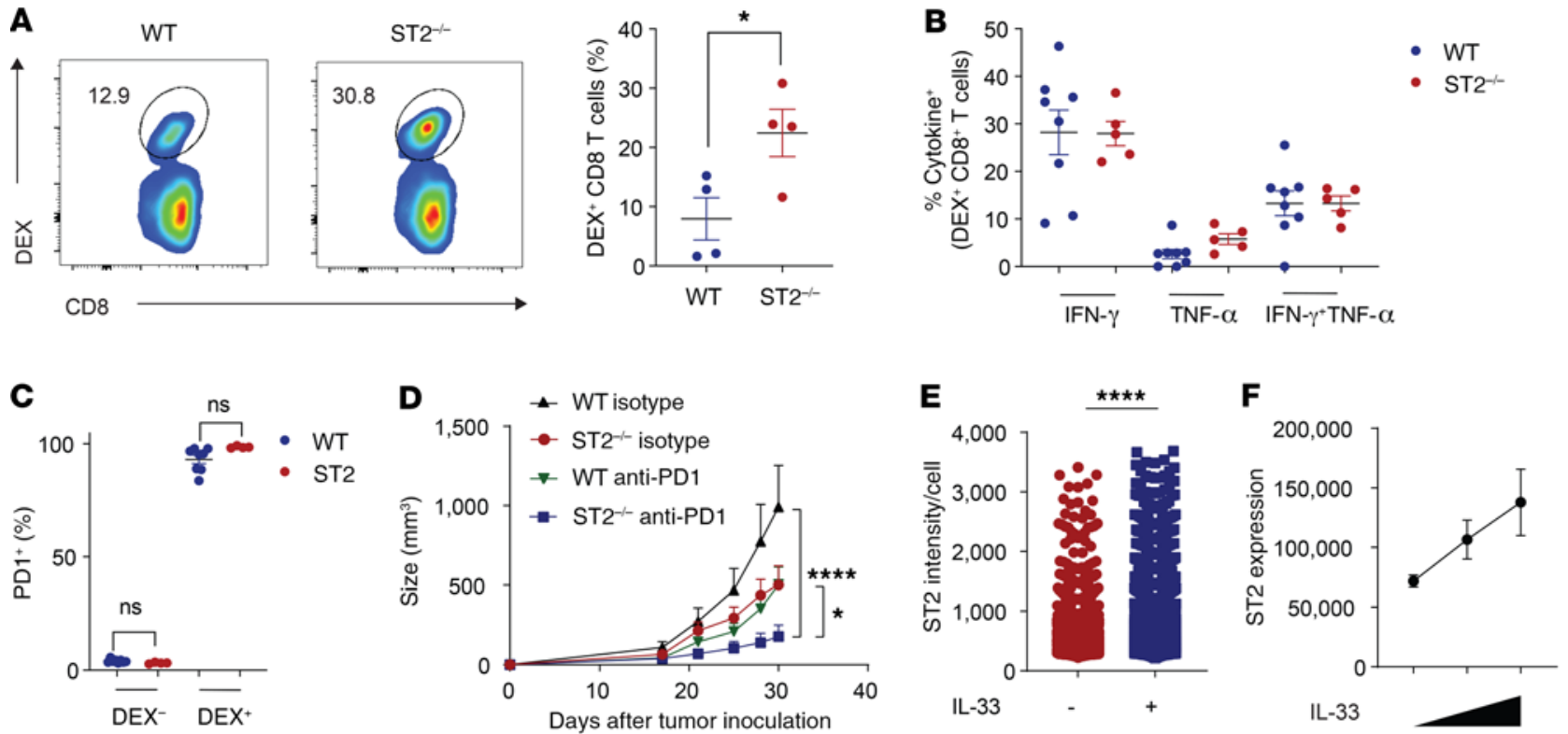

G

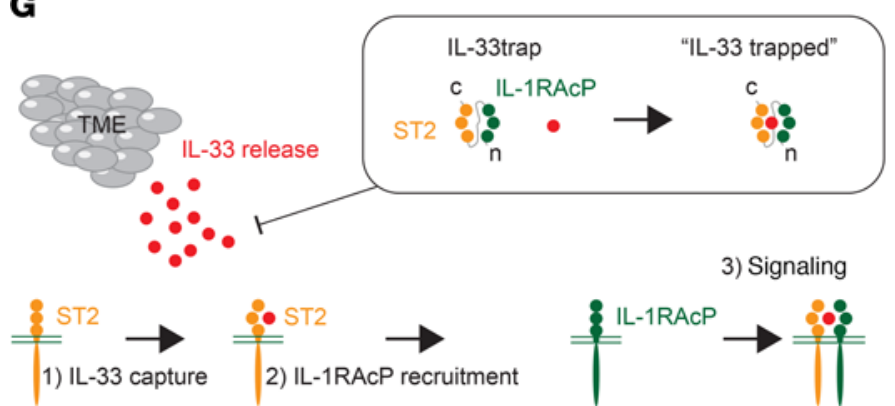

H

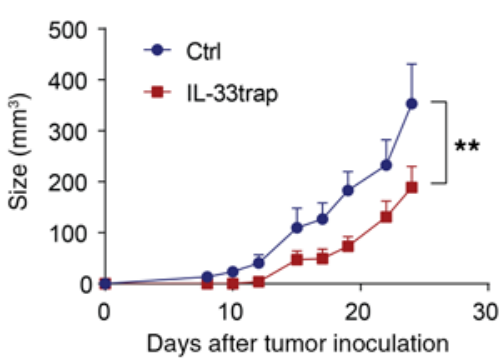

I

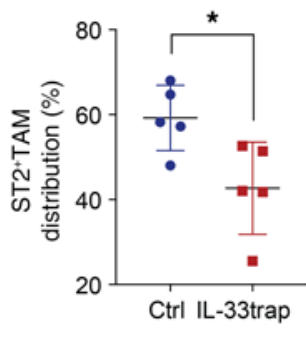

Figure 5. Inhibition of the IL-33/ST2 pathway on antigen-specific T cell responses. (A-C) Injection of splenic CD8-enriched OT-I T cells into WT or $S T 2^{-1-}$ mice (day 0). Mice were vaccinated intraperitoneally with $100 \mu \mathrm{g}$ SIINFEKL peptide plus $100 \mu \mathrm{g}$ poly(I:C). (A) The number of dextramer-positive (DEX) CD8 ${ }^{+}$T cells was determined from splenocytes on day 5 (representative of 2 independent experiments; $n=4$ mice/group; data displayed as mean + SEM). (B) Percentages of IFN- $\gamma$ - and TNF- $\alpha$ - expressing cells were determined upon SIINFEKL peptide restimulation (WT, $n=8 ; 5 T 2^{-/}, n=5 ;$ data displayed as mean + SEM). (C) Expression of PD-1 in vaccinated mice is shown (WT, $n=8 ; S T 2^{-1-}, n=4$; data displayed as mean + SEM). (D) Therapeutic efficacy of anti-PD-1 treatment in WT and ST2 ${ }^{-/-}$mice in the subcutaneous MC38 CRC model (WT isotype, $n=9 ; 5 T 2^{-1-}, n=7 ;$ WT anti-PD-1, $n=11$; $S T 2^{-1-}$ anti-PD-1, $n=10$; data displayed as mean + SEM). (E) MACS F4/80-sorted macrophages from WT mice bearing MC38 tumors were seeded and treated or not with IL-33. ST2 intensity per cell was determined using confocal microscopy. Graph depicts the intensity of ST2 per cell (data displayed as mean + SD). Without IL33, $n=2793$; with IL-33, $n=4217$. (F) Dose-dependent increase in ST2 expression upon IL-33 stimulation ( $n=5$; data displayed as mean + SEM). (G) Schematic representation of the IL-33trap. (H) Therapeutic efficacy of IL-33trap treatment in WT mice harboring MC38 tumors (control [CtrI], $n=6$, IL-33trap, $n=5$; data displayed as mean + SEM). (I) Analysis of ST2 ${ }^{+}$TAM infiltration from control and IL-33trap-treated tumors ( $n$ $=5$; data displayed as mean + SD). ${ }^{*} P<0.05,{ }^{* *} P<0.01,{ }^{* * *} P<0.0001$. Significance was determined by 2-tailed unpaired $t$ test (A, C, E, and I), 1-way ANOVA (B) and 2-way ANOVA (D and $\mathbf{H})$.

\section{Methods}

Mice and cell lines. C57BL/ 6 and BALB/c mice, 6-8 weeks old, female and male, were purchased from the Jackson Laboratory and housed under pathogen-free conditions. OT-I mice contained transgenic inserts for mouse Tcra-V2 and Tcrb-V5 genes. The transgenic T cell receptor was designed to recognize the ovalbumin peptide SIINFEKL $\left(\mathrm{OVA}_{257-264}\right)$ in the context of $\mathrm{H}-2 \mathrm{~Kb}$. These mice were initially purchased from

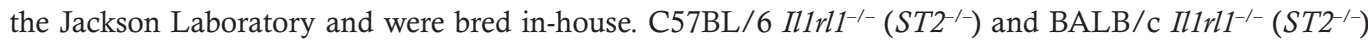

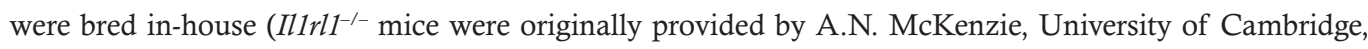
Cambridge, United Kingdom) and matched for age and sex to the WT mice.

MC38 cells (colon carcinoma, MSI model, C57BL/6) were obtained from Patrick Hwu, MD Anderson Cancer Center, Houston, Texas, USA), and CT26 cells (colon carcinoma, MSS model, BALB/c) were obtained from ATCC (31). MC38 cells were maintained in DMEM supplemented with 10\% FBS (MilliporeSigma) and $1 \%$ penicillin-streptomycin solution $(10,000 \mathrm{U} / \mathrm{ml}$ penicillin, $10,000 \mu \mathrm{g} / \mathrm{mL}$ streptomycin; 
HyClone). CT26 cells were maintained in RPMI medium supplemented with 10\% FBS (MilliporeSigma) and $1 \%$ penicillin-streptomycin solution $(10,000 \mathrm{U} / \mathrm{ml}$ penicillin, $10,000 \mu \mathrm{g} / \mathrm{mL}$ streptomycin; HyClone). Cell lines were cultured at $37^{\circ} \mathrm{C}$ in a humidified incubator, $5 \% \mathrm{CO}_{2}$.

Tumor implantation and antibody treatment. In the subcutaneous setting, $1 \times 10^{5} \mathrm{MC} 38$ cells or $5 \times 10^{4}$ CT26 cells were injected in $50 \mu \mathrm{L}$ PBS subcutaneously into the right flank. Tumor growth was followed and measured using a digital caliper 3 times per week. The tumor volume was calculated as smallest tumor size $\times$ smallest tumor size $\times$ largest tumor size divided by 2 . For the orthotopic injection of $1 \times 10^{5}$ MC38 cells in the cecum, mice were anesthetized and shaved at the belly area. A small incision of the skin and muscle layer was performed to expose the cecum. MC38 cells were injected in a final volume of $50 \mu \mathrm{L}$ PBS using a 30 gauge needle. The cecum was repositioned in the mice, and the wound was closed using surgical sutures and wound clips.

In the therapeutic setting, mice were treated with anti-PD-1 (200 $\mu \mathrm{g}$; clone RMP1-14; Bio X Cell) administered i.p. as indicated in the treatment schedule. For depletion experiments anti-CSF1R (400 $\mu \mathrm{g}$; clone AFS98; Bio X Cell) was administered 1 week prior to tumor cell injection and the injection continued until the end of the experiment. CD ${ }^{+} \mathrm{T}$ cells were depleted using anti-CD8 (100 $\mu$; clone 53-6.72, Bio X Cell) was initiated 2 days prior to tumor cell inoculation and the injection continued until the end of the experiment. The rat IgG2a isotype control (clone 2A3, Bio X Cell) was administered at the same dose as the depletion or therapeutic antibodies in the indicated experiments. The anti-CSF1R, anti-CD8, and isotype antibodies were administered 3 times per week. The dilution of the antibodies was done using $\mathrm{pH} 7.0$ dilution buffer (Bio X Cell). IL-33trap was produced at the Protein Core of the VIB as described previously (41). In the IL-33trap experiment, mice were injected subcutaneously with $1 \times 10^{5} \mathrm{MC} 38$ cells and on the same day injected for the first time with $50 \mu \mathrm{g}$ IL-33trap in a volume of $100 \mu \mathrm{L}$ PBS. Control treated mice were administered $100 \mu \mathrm{L}$ PBS on a daily basis. Mice were treated daily until the end of the experiment.

In vivo T cell response assay. For the in vivo $\mathrm{T}$ cell response assay, $1 \times 10^{6}$ enriched OT-I CD $8^{+} \mathrm{T}$ cells were injected i.v. in $200 \mu \mathrm{L}$ PBS into the mice on day 1. The purity of the enriched cells was $>90 \%$. On day 0 , mice were i.p. vaccinated with $100 \mu \mathrm{g}$ SIINFEKL peptide (MilliporeSigma) together with $100 \mu \mathrm{g}$ poly(I:C) (Invivogen). Antigen-specific immune responses were evaluated upon analysis of blood and spleen using flow cytometry. To detect IFN- $\gamma$ and TNF- $\alpha$ expression on antigen-specific T cells, cells were restimulated overnight with $5 \mu \mathrm{g} / \mathrm{mL}$ SIINFEKL peptide. After 2 hours of peptide restimulation, brefeldin A (1:1000; BioLegend) was added to the samples.

Macrophage suppression assay. Single-cell suspensions from spleens and tumors were prepared as described above. Enriched naive pan-T cells (130-095-130, Miltenyi Biotec) were then labeled with $10 \mu \mathrm{M}$ CFSE (BioLegend) for 10 minutes at $37^{\circ} \mathrm{C}$. Thereafter, the cells were neutralized and washed twice in culture medium containing $10 \%$ FBS. The pan-T cells $\left(1 \times 10^{5}\right.$ cells $)$ were then cocultured with enriched F4/80+ tumor macrophages (130-110-443, Miltenyi Biotec) and stimulated using $1 \mu \mathrm{L}$ antiCD3/CD28 Dynabeads (Gibco). The cells were cultured in a 96-well U-bottom plate with a final volume of $200 \mu \mathrm{L}$ medium containing IL-2 (2 ng/mL; 212-12, Peprotech) with and without IL-33 (10 ng/ $\mathrm{mL}$; R\&D Systems), as indicated. CFSE proliferation of the T cells was assessed using flow cytometry and cytokine release using IFN- $\gamma$ ELISA (BioLegend) on the coculture supernatants.

Tumor dissociation and isolation of mouse cells. Single-cell suspensions from MC38 and CT26 tumors were prepared using the mouse Tumor Dissociation Kit (130-096-730, Miltenyi Biotec) according to the manufacturer's guidelines. Macrophages were enriched from single-cell suspensions from MC38 and CT26 tumors using mouse anti-F4/80 MicroBeads UltraPure (130-110-443, Miltenyi Biotec). To enrich $\mathrm{T}$ cells, spleens were resected and crushed with the back of a 3-mL syringe over a $40-\mu \mathrm{m}$ filter. After RBC lysis, using RBC Lysis Buffer (BioLegend), the cell suspensions were further enriched using mouse CD8a (Ly-2) MicroBeads (130-117-044, Miltenyi Biotec) or mouse Pan T Cell Isolation Kit II (130-095-130, Miltenyi Biotec). Analysis of blood cells was done after lysis of the RBCs using the RBC Lysis Buffer.

Flow cytometry. Acquisition was performed using an LSR Fortessa X-20 or LSR Fortessa (BD Biosciences), and data analysis was done using FlowJo version 10. Live/dead status was assessed using SYTOX Blue (Invitrogen) or eBioscience Fixable Viability Dye eFluor 506 (Invitrogen). Blocking of a specific signal was done using 10\% normal goat serum, 10\% FBS, and/or preincubation with CD16/32 (BioLegend). For staining including cell permeabilization, the cells were fixed and permeabilized using the BD Cytofix/Cytoperm fixation permeabilization kit (BD Biosciences). The following antibodies 
were used in several combinations: CD3-PE/Cy7 (1:100; clone 17A2, BioLegend), CD4-AF700 (1:100; clone GK1.5, BioLegend), CD8a-APC/Cy7 (1:100; clone 53-6.7, BioLegend), CD11b-PE (1:200, clone M1/70; BioLegend), CD11b-eFluor 450 (1:200; clone M1/70; Invitrogen), CD11c-AF647 (1:200; clone N418, BioLegend), CD25-PerCP/Cy5.5 (1:200; clone 3C7, BioLegend), CD25-APC (1:200; clone 3C7, BioLegend), F4/80-PerCPCy5.5 (1:100; clone 3C7, BioLegend), CD45-BV605 (1:100; clone 30-F11, BioLegend), CD45-V500 (1:100; clone 30-F11, BD Biosciences), CD127-PE/Dazzle594 (1:100; clone A7R34, BioLegend), CD274-BV421 (1:100; clone 10F.9G2, BioLegend), CD279-BV421 (1:50; clone 29F.1A12, BioLegend), F4/80-PerCP/Cy5.5 (1:100; clone BM8, BioLegend), FOXP3-AF647 (1:100; clone MF-14, BioLegend), I-A/I-E-BV421 (1:1000; clone M5/114.15.2, BioLegend), Ly-6C-AF700 (1:300; clone HK1.4, BioLegend), Ly-6G-APC/Cy7 (1:300; clone 1A8, BioLegend), CD19-BV656 (1:200; clone 6D5, BioLegend), GR-1-FITC (1:200; clone RB6-865, BioLegend), ST2-PE (1:200; clone DJ8, MD Biosciences), TCR- $\beta-\mathrm{PE} / \mathrm{Cy} 7$ (1:100; clone H57-597, BioLegend), IgG1-PE (1:200; clone P3.6-2.8.1, Invitrogen), TNF- $\alpha-P E$ (1:50; clone MP6-XT22, BioLegend), IFN- $\gamma-B V 786$ (1:50; clone XMG1.2, BD Biosciences), CXCR3-PE/Cy7 (1:50; clone CXCR3-173; BioLegend), SIRPa-PE/Dazzle 594 (1:500; clone P84, BioLegend), CD169-BV605 (1:1000; clone 3D6.112, BioLegend), CD206-BV650 (1:500; clone C068C2, BioLegend), PD-L1-BV785 (1:200; clone 10F.9G2, BioLegend), MHC Dextramer H-2Kb-SIINFEKL-APC (10 $\mu \mathrm{L}$; JD2163, Immudex).

Mass cytometry. Single tumor cells were obtained as described above and washed twice using freshly prepared in-house CyTOF buffer (cold PBS containing 0.5\% BSA and 0.02\% azide). For each sample, $1.5 \times 10^{6}$ cells were used. The cells were stained with the antibody panel described in Supplemental Table 2. CyTOF data were evaluated via viSNE analysis using the Cytobank platform (42). viSNE analysis allows the visualization of a high-dimensional analysis in 2 dimensions using the Barnes-Hut implementation of the t-distributed stochastic neighbor embedding (tSNE) algorithm. We ran the viSNE analysis on the samples through a proportional sampling, with 7500 iterations, a perplexity of 30 , and a $\theta$ of 0.5 . On this viSNE analysis we performed SPADE clustering (43). The cell populations were then manually gated on the SPADE tree based on the selected markers. Thereafter, these clustered cell populations were visualized on viSNE as an overlaid plot.

Colon cancer transcriptomics data analysis. For the bioinformatics analysis, we used CRC data sets from the The Cancer Genome Atlas (TCGA) database (456 colon adenocarcinoma [COAD] patients), and from the NCBI's Gene Expression Omnibus database (GSE41258, 165 CRC patients; and GSE39582, 505 CRC patients). We divided the patients into IL1RL1 high- and low-expression groups by the top and bottom $40 \%$ of the expression level of the gene. Survival curves of the IL1RL1-high and -low patients were tested by log-rank test by using the "survival" R package on the GSE41258 and GSE39582 data sets. TCGA COAD RNA-Seq data were retrieved from the Genomic Data Commons Data Portal with gene expression level normalized by fragments per kilobase of transcript per million (FPKM). We utilized our recently developed deconvolution method, ICTD (9), to access proportions of T cells and other immune and stromal cell types in each patient. Specifically, ICTD identifies data set-specific cell type uniquely expressing gene markers to optimize the estimation of cell proportion. In the TCGA COAD data set, we identified a high coexpression correlation among $C D 2, C D 3 D, C D 3 E$, and $C D 3 G$ genes. The first eigenvector of the expression profile of these genes was used to estimate the proportion of total $\mathrm{T}$ cells in each sample. We further identified that the T cell cytotoxicity marker genes, namely, CD8A, SLA2, NKG7, $P R F 1, G Z M A$, and $G Z M H$, were highly coexpressed in the samples with high predicted level of total $\mathrm{T}$ cells (93 patients in total: IL1RL1-high, 53 patients; IL1RL1-low, 40 patients). The first eigenvector of the expression profile of these genes were used to estimate the whole tissue cytotoxicity level in the samples with high $\mathrm{T}$ cell levels. Relative cytotoxicity level was computed by the following formula: relative cytotoxicity levels $=$ whole tissue cytotoxicity level/total $\mathrm{T}$ cell proportion .

Microarray data analysis. We systematically evaluated the expression level of ST2 in different cell types by using a large collection of microarray data of different cell types. All data were generated by using Affymetric UA133 Plus 2.0 Array on purified bulk cell samples. Cell types analyzed include the following, with datasets and samples shown for each type: fibroblasts, 34, 387; adipocytes, 3, 26; B cells, 20, 404; $\mathrm{CD}^{+} \mathrm{T}$ cells, 23, 443; CD8 ${ }^{+} \mathrm{T}$ cells, 9, 130; dendritic cells, 32, 410; and macrophages, $21,277$. Detailed sample information is available in Supplemental Table 3. All data were normalized by using MAS5, with batch effect removed by using ComBat and quantile normalization $(44,45)$. The normalized expression level of ST2 in each cell type was plotted as shown in Figure 1B. 
Confocal microscopy and analysis. Confocal imaging was conducted using an Olympus FV1000 MPE confocal/2-photon system equipped with an Olympus UPlanSApo $\times 60 / 1.2$ objective lens, at the Indiana Center for Biological Microscopy (ICBM) Imaging Facility, Indiana University. Images were collected at a frame size of $2048 \times 2048$ pixels $(210.8 \times 210.8 \mu \mathrm{m}), 8 \mu \mathrm{s} /$ pixel scanning rate, and a sequential illumination scanning mode set up for 3 channels: blue (405-nm excitation/425- to 475-nm emission), green (488-nm excitation/500- to 550-nm emission), and red (635-nm excitation/655- to 755-nm emission). To ensure that measurements would be comparable between different specimens, all images were collected at the same scanning settings. Four to 7 representative fields per sample were acquired. For confocal immunofluorescence microscopy, we used the following antibodies: ST2L (101002, MD Biosciences), ST2 (101001, MD Biosciences), CD68 (76437, Cell Signaling Technology), and F4/80 (70076S, Cell Signaling Technology).

Quantitative measurements were performed using Imaris software (Bitplane). The total number of cells per image was calculated based on a blue/DAPI channel, using a "Spots" segmentation module. The green and red channels, corresponding to ST2 and CD68 staining, respectively, were segmented using the Imaris "Surfaces" module. A subset of the total number of nuclei was then selected to indicate the tumor stroma. Based on a "Spot-Surfaces" distance analysis, red- and green-positive cells among all stroma cells were

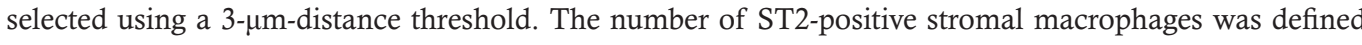
using the same approach.

Digital whole slide imagining and Aperio image analysis software. Tissue slides were imaged using Aperio Scanscope CS. Computer-assisted morphometric analysis of digital images was performed using the positive pixel algorithm from Aperio Image Analysis software. For immunohistochemistry, we used the following antibodies: CD8 $\alpha$ (98941S, Cell Signaling Technology) and F4/80 (70076S, Cell Signaling Technology) and stained 3- $\mu \mathrm{m}$-thick formalin-fixed paraffin-embedded sections as described previously (46).

Statistics. All statistics (average values, SD, SEM, significant differences between groups) were calculated using GraphPad Prism 7 and R. Evaluation of 2 data sets was done using 2-tailed unpaired Student's $t$ test, while the evaluation of more than 2 groups was done using 1-way or 2-way ANOVA as indicated. The graphs display the results as mean \pm SD or mean \pm SEM. Survival curves are visualized as Kaplan-Meier plots and analyzed using the log-rank test. $P$ values less than 0.05 were considered significant.

Study approval. All mice were housed in the animal facility of Indiana University, and all procedures were carried with approval of the Indiana University Institutional Animal Care and Use Committee.

\section{Author contributions}

KVDJ, SP, and XL conceived, coordinated, and designed the study. KVDJ, YF, ZZ, TY, MMK, YL, HE, MF, JY, HJ, XH, and XZ performed experiments. KVDJ, YS, KS, CZ, and XL analyzed the bioinformatics data. KVDJ and MMK analyzed the microscopy data. GES analyzed the tissue toxicity from H\&E stainings. $\mathrm{RB}$ and $\mathrm{HB}$ produced and provided the IL-33trap. KVDJ, SP, and XL interpreted the results and wrote the manuscript with input from all authors.

\section{Acknowledgments}

We thank A.N. McKenzie for providing the $I l 1 r l 1^{-/-}$mice and $\mathrm{H}$. Xu for technical assistance. We thank the Melvin and Bren Simon Cancer Center at Indiana University School of Medicine for use of the Tissue Procurement \& Distribution Core, which provided histology slides service. The authors thank the members of the Melvin and Bren Simon Cancer Center Flow Cytometry Resource Facility (FCRF) for outstanding technical support. The FCRF is funded in part by NIH grants P30CA082709, U54 DK106846, and 1S10D012270. The study is supported in part by the IUSM Strategic Research Initiative fund; NIH R01CA203737 to X. Lu; and NIH U01CA232491 to S. Paczesny.

Address correspondence to: Sophie Paczesny, Melvin and Bren Simon Cancer Center, Wells Center for Pediatric Research, Indiana University School of Medicine, 1044 W Walnut Street, R4 425, Indianapolis, Indiana 46202, USA. Phone: 317.278.5487; Email: sophpacz@iu.edu. Or to: Xiongbin Lu, Vera Bradley Foundation Chair in Breast Cancer Innovation, Indiana University Melvin and Bren Simon Cancer Center, Indiana University School of Medicine, 980 W Walnut St, R3.C218D, Indianapolis, Indiana, USA. Phone: 46202, 317.274.4398. Email: xiolu@iu.edu. 
1. Van der Jeught K, Xu HC, Li YJ, Lu XB, Ji G. Drug resistance and new therapies in colorectal cancer. World J Gastroenterol. 2018;24(34):3834-3848.

2. Van der Jeught $\mathrm{K}$, et al. Targeting the tumor microenvironment to enhance antitumor immune responses. Oncotarget. 2015;6(3):1359-1381.

3. Sanmamed MF, Chen L. A paradigm shift in cancer immunotherapy: from enhancement to normalization. Cell. 2018;175(2):313-326.

4. Popovic A, Jaffee EM, Zaidi N. Emerging strategies for combination checkpoint modulators in cancer immunotherapy. J Clin Invest. 2018;128(8):3209-3218.

5. Cayrol C, Girard JP. IL-33: an alarmin cytokine with crucial roles in innate immunity, inflammation and allergy. Curr Opin Immunol. 2014;31:31-37.

6. Pastille E, et al. The IL-33/ST2 pathway shapes the regulatory T cell phenotype to promote intestinal cancer. Mucosal Immunol. 2019;12(4):990-1003.

7. Schiering C, et al. The alarmin IL-33 promotes regulatory T-cell function in the intestine. Nature. 2014;513(7519):564-568

8. Ameri AH, et al. IL-33/regulatory T cell axis triggers the development of a tumor-promoting immune environment in chronic inflammation. Proc Natl Acad Sci U S A. 2019;116(7):2646-2651.

9. Chang W, et al. ICTD: inference of cell types and deconvolution - a next generation deconvolution method for accurate assess cell population and activities in tumor microenvironment. bioRxiv. 6593;2018:426593.

10. Andrews LP, Marciscano AE, Drake CG, Vignali DA. LAG3 (CD223) as a cancer immunotherapy target. Immunol Rev. 2017;276(1):80-96.

11. Grosso JF, et al. LAG-3 regulates $\mathrm{CD}^{+} \mathrm{T}$ cell accumulation and effector function in murine self- and tumor-tolerance systems J Clin Invest. 2007;117(11):3383-3392.

12. Delacher M, et al. Genome-wide DNA-methylation landscape defines specialization of regulatory T cells in tissues. Nat Immunol. 2017;18(10):1160-1111.

13. Xu D, et al. Selective expression of a stable cell surface molecule on type 2 but not type 1 helper T cells. J Exp Med. 1998;187(5):787-794.

14. Hatzioannou A, et al. An intrinsic role of IL-33 in $\mathrm{T}_{\text {reg }}$ cell-mediated tumor immunoevasion. Nat Immunol. 2020;21(1):75-85.

15. Andersson P, et al. Molecular mechanisms of IL-33-mediated stromal interactions in cancer metastasis. JCI Insight. 2018;3(20):122375.

16. Yang Y, et al. The PDGF-BB-SOX7 axis-modulated IL-33 in pericytes and stromal cells promotes metastasis through tumour-associated macrophages. Nat Commun. 2016;7:11385.

17. Qian B, et al. A distinct macrophage population mediates metastatic breast cancer cell extravasation, establishment and growth. PLoS ONE. 2009;4(8):e6562.

18. Cassetta L, Pollard JW. Targeting macrophages: therapeutic approaches in cancer. Nat Rev Drug Discov. 2018;17(12):887-904.

19. Kitamura T, et al. CCL2-induced chemokine cascade promotes breast cancer metastasis by enhancing retention of metastasis-associated macrophages. J Exp Med. 2015;212(7):1043-1059.

20. Butler KL, Clancy-Thompson E, Mullins DW. CXCR3 ${ }^{+}$monocytes/macrophages are required for establishment of pulmonary metastases. Sci Rep. 2017;7:45593.

21. Holgado A, et al. IL-33trap is a novel IL-33-neutralizing biologic that inhibits allergic airway inflammation. J Allergy Clin Immunol. 2019;144(1):204-215.

22. Ishida Y, Agata Y, Shibahara K, Honjo T. Induced expression of PD-1, a novel member of the immunoglobulin gene superfamily, upon programmed cell death. EMBO J. 1992;11(11):3887-3895.

23. Leach DR, Krummel MF, Allison JP. Enhancement of antitumor immunity by CTLA-4 blockade. Science. 1996;271(5256):1734-1736.

24. Kim TK, Herbst RS, Chen L. Defining and understanding adaptive resistance in cancer immunotherapy. Trends Immunol. 2018;39(8):624-631.

25. Couzin-Frankel J. Breakthrough of the year 2013. Cancer immunotherapy. Science. 2013;342(6165):1432-1433.

26. Wang J, et al. Siglec-15 as an immune suppressor and potential target for normalization cancer immunotherapy. Nat Med. 2019;25(4):656-666.

27. Fujiyoshi K, et al. Metastatic pattern of stage IV colorectal cancer with high-frequency microsatellite instability as a prognostic factor. Anticancer Res. 2017;37(1):239-247.

28. Merok MA, et al. Microsatellite instability has a positive prognostic impact on stage II colorectal cancer after complete resection: results from a large, consecutive Norwegian series. Ann Oncol. 2013;24(5):1274-1282.

29. de la Chapelle A, Hampel H. Clinical relevance of microsatellite instability in colorectal cancer. J Clin Oncol. 2010;28(20):33803387.

30. Popat S, Hubner R, Houlston RS. Systematic review of microsatellite instability and colorectal cancer prognosis. J Clin Oncol. 2005;23(3):609-618.

31. Efremova M, et al. Targeting immune checkpoints potentiates immunoediting and changes the dynamics of tumor evolution. Nat Commun. 2018;9(1):32.

32. Fang M, et al. IL33 promotes colon cancer cell stemness via JNK activation and macrophage recruitment. Cancer Res. 2017;77(10):2735-2745.

33. Zhiguang X, et al. Over-expression of IL-33 leads to spontaneous pulmonary inflammation in mIL-33 transgenic mice. Immunol Lett. 2010;131(2):159-165.

34. Bonilla WV, et al. The alarmin interleukin-33 drives protective antiviral CD8 ${ }^{+}$T cell responses. Science. 2012;335(6071):984-989

35. Ramadan A, et al. Specifically differentiated T cell subset promotes tumor immunity over fatal immunity. JExp Med. 2017;214(12):3577-3596.

36. Griesenauer B, et al. ST2/MyD88 deficiency protects mice against acute graft-versus-host disease and spares regulatory T cells. J Immunol. 2019;202(10):3053-3064.

37. Kurowska-Stolarska M, et al. IL-33 amplifies the polarization of alternatively activated macrophages that contribute to airway 
inflammation. J Immunol. 2009;183(10):6469-6477.

38. Rank MA, Kobayashi T, Kozaki H, Bartemes KR, Squillace DL, Kita H. IL-33-activated dendritic cells induce an atypical TH2type response. J Allergy Clin Immunol. 2009;123(5):1047-1054.

39. Ramadan AM, et al. From proteomics to discovery of first-in-class ST2 inhibitors active in vivo. JCI Insight. 2018;3(14):99208.

40. Castle JC, et al. Immunomic, genomic and transcriptomic characterization of CT26 colorectal carcinoma. BMC Genomics. 2014; $15: 190$.

41. Holgado A, Braun H, Van Nuffel E, Detry S, Schuijs MJ, Deswarte K, et al. IL-33trap is a novel IL-33-neutralizing biologic that inhibits allergic airway inflammation. J Allergy Clin Immunol. 2019;144(1):204-215.

42. Amir el-AD, et al. viSNE enables visualization of high dimensional single-cell data and reveals phenotypic heterogeneity of leukemia. Nat Biotechnol. 2013;31(6):545-552.

43. Qiu P, et al. Extracting a cellular hierarchy from high-dimensional cytometry data with SPADE. Nat Biotechnol. 2011;29(10):886-891.

44. Pepper SD, Saunders EK, Edwards LE, Wilson CL, Miller CJ. The utility of MAS5 expression summary and detection call algorithms. BMC Bioinformatics. 2007;8:273.

45. Leek JT, Johnson WE, Parker HS, Jaffe AE, Storey JD. The sva package for removing batch effects and other unwanted variation in high-throughput experiments. Bioinformatics. 2012;28(6):882-883.

46. Li Y, et al. Heterozygous deletion of chromosome $17 \mathrm{p}$ renders prostate cancer vulnerable to inhibition of RNA polymerase II. Nat Commun. 2018;9(1):4394. 